\title{
Burnout-Symptomatik bei KlinikärztInnen
}

\author{
Ilsemarie Kurzthaler · Georg Kemmler · W. Wolfgang Fleischhacker
}

Eingegangen: 23. Februar 2017 / Angenommen: 25. April 2017 / Online publiziert: 23. Mai 2017

(C) Der/die Autor(en) 2017. Dieser Artikel ist eine Open-Access-Publikation.

\section{Zusammenfassung}

Hintergrund Burnout ist kein akuter Zustand sondern eine anhaltende Stressreaktion, gekennzeichnet durch emotionale Erschöpfung, Depersonalisation und fehlende persönliche Erfüllung. Ziel dieser Studie war es, Prävalenz und Ausprägung der Burnout-Symptomatik in einer Population von KlinikärztInnen zu untersuchen und einen möglichen Zusammenhang zwischen Burnout-Symptomatik und soziodemografischen und berufsbezogenen Variablen zu ermitteln.

Methodik 69 an der Universitätsklinik bzw. am Landeskrankenhaus Innsbruck beschäftigte ÄrztInnen unterschiedlicher Fachrichtungen $\leq 55$ Jahre wurden in diese Querschnittsuntersuchung eingeschlossen. Neben soziodemografischen und berufsbezogenen Daten wurde mit Hilfe des Maslach Burnout Inventory (MBI) die Burnout-Symptomatik erhoben.

Ergebnisse Die Einteilung nach Burnout-Risiko aufgrund der Kombinationen der drei MBI-Subskalen ergab für 8,8\% der KlinikärztInnen ein hohes Risiko. Diese ÄrztInnen waren durch starke emotionale Erschöpfung, starke oder mittelgradige Depersonalisation und geringe bzw. in einem Fall mäßige persönliche Erfüllung charakterisiert. Weitere $11,8 \%$ der teilnehmenden ÄrztInnen zeigten ein mäßiges Burnout-Risiko. Soziodemografische Variablen, Ausbildungsstand und Fachrichtung zeigten keinen signifikanten Einfluss auf die Burnout-Symptomatik. Dagegen korrelierte wissenschaftliche Tätigkeit mit einer signifikant

a.Univ.-Prof. Dr. med.univ. I. Kurzthaler $(\bowtie)$.

Priv.-Doz. Dr. rer.nat. G. Kemmler .

Univ.-Prof. Dr. med.univ. W. W. Fleischhacker

Department für Psychiatrie, Psychotherapie

und Psychosomatik, Universitätsklinik für

Psychiatrie I, Medizinische Universität Innsbruck,

Anichstraße 35, 6020 Innsbruck, Österreich

ilsemarie.kurzthaler@i-med.ac.at höheren persönlichen Erfüllung und erwies sich somit als protektiver Faktor im Rahmen der BurnoutEntwicklung.

Fazit für die Praxis Diese Untersuchung zeigt, dass Burnout-Symptomatik bei KlinikärztInnen unabhängig von der Fachrichtung eine erhebliche Problematik darstellt. Die Entwicklung wissenschaftlich fundierter Maßnahmen im Sinne der Burnoutprävention und -therapie ist daher erforderlich.

Schlüsselwörter Burnout · KlinikärztInnen · BurnoutRisiko · Burnout-Gefährdung

\section{Burnout in physicians}

\section{Summary}

Background Burnout is a syndrome characterized by emotional exhaustion, depersonalization and low personal accomplishment. The primary objective of this study was to investigate both the prevalence and severity of burnout symptoms in a sample of clinical physicians from different speciality disciplines.

Methods A total of 69 clinical physicians $\leq 55$ years who are working at the Medical University/regional Hospital Innsbruck were included into a cross-sectional study. Next to the assessment of sociodemographic and work-related variables the Maslach Burnout Inventory (MBI) was used to investigate burnout symtoms.

Results Overall, $8.8 \%$ of the study population showed high emotional exhaustion with high or moderate depersonalization and low personal accomplishment and therefore had a high risk to develop a burnout syndrom. $11.8 \%$ showed a moderade burnout risk. Neither sociodemographic variables nor the degree of educational qualification or speciality discipline had an influence on burnout symptoms. However, there 
was a positive correlation between scientific activity and personal accomplihment.

Conclusion Our results suggest that the dimension of burnout symtoms among clinical physicians in Austria has be taken seriously. Further research is needed to develop specific programs in terms of burnout prevention and burnout therapy.

Keywords Burnout - Clinical physicians · Burnout risk · Burnout endangerment

\section{Hintergrund}

Die Problematik eines allgemeinen ÄrztInnenmangels in Österreich [1] wird seit Jahren diskutiert, gewinnt aber aufgrund demographischer Entwicklungen sowohl der Bevölkerung als auch der Ärzteschaft immer mehr an Aktualität. Gesunde, gut ausgebildete Ärztinnen und Ärzte mit hoher Arbeitsmotivation sind von essentieller Bedeutung für ein gut funktionierendes Gesundheitssystem. Die Arbeitsmotivation hat konkrete Auswirkungen darauf, wie gut oder engagiert jemand seine Arbeit erledigt [2]. Cassel und Jain nennen als intrinsische Motivationsfaktoren zum ärztlichen Handeln vor allem Leistungsorientierung, Befriedigung durch die Bewältigung schwieriger Aufgaben, Befriedigung durch das Erzielen guter klinischer Ergebnisse, Autonomie, Respekt und kollegiale Beziehungen. Als extrinsische Motivationsfaktoren nennen sie zusätzlich hohe Vergütung und Anerkennung durch die Patienten [3].

Im Rahmen der ÄrztInnengesundheit wird aktuell das Thema Burnout intensiv diskutiert. Zahlreiche Publikationen und Beiträge in den verschiedensten Medien greifen diese Problematik auf. Schätzungsweise sind schon über 6000 Bücher, Kapitel, Dissertationen und Zeitschriftenartikel zum Thema Burnout veröffentlicht worden [4]. Trotz des Interesses an dieser Thematik gibt es bis heute noch keine allgemeingültige Definition dieses Begriffes. Allgemein anerkannt ist jedoch die Beschreibung von Burnout als Risikozustand, der sich aufgrund chronischer berufsbezogener Stresssituationen entwickeln kann und durch drei Kernsymptome gekennzeichnet ist: emotionale Erschöpfung, Depersonalisation und geringe persönliche Erfüllung. Aus der Erschöpfung heraus distanzieren sich die Betroffenen auf emotionale und kognitive Weise von ihrer Arbeit und den Menschen, mit denen sie im Rahmen ihrer Berufsausübung zu tun haben (Depersonalisation). Parallel dazu entwickelt sich ein Gefühl der reduzierten Effektivität oder Leistungsfähigkeit verbunden mit einer geringen persönlichen Erfüllung [5].

Im ICD-10 wurde 1991 erstmals der Begriff Burnout aufgeführt [6]. Burnout wird definiert als Ausgebranntsein und findet sich unter $\mathrm{Z} 73.0$ und damit im Kapitel „Probleme mit Bezug auf Schwierigkeiten bei der Lebensbewältigung“ [7]. Burnout stellt damit allerdings keine eigenständige Diagnose dar. Burnout hat einen gewissen Krankheitswert, ,ist aber keine anerkannte somatische oder psychiatrische Krankheit“ [8]. Im Unterschied zur Depression oder depressiven Anpassungsstörung bezieht sich der Begriff Burnout ausschließlich auf die individuelle Beziehung zum eigenen Arbeitsumfeld.

Shanafelt et al. [9] kennzeichnen das Phänomen Burnout als ein gerade im medizinischen Arbeitsfeld häufig anzutreffendes. Michalsen und Hillert [10] beschreiben bei in der Anästhesie und Intensivmedizin tätigen ÄrztInnen eine Punktprävalenz für ein mittleres oder hohes Burnout-Niveau, gemessen mit dem Maslach Burnout Inventory (MBI) [11], von etwa 40-50\%. und laut Reimann [12] weist jeder dritte Arzt unabhängig von seiner Fachrichtung über die Lebens(respektive Berufs-)zeit Symptome eines Burnout auf.

Im Jahr 2006 untersuchten Kinzl et al. mittels MBI, bei 89 AnästhesistInnen und IntensivmedizinerInnen der Universitätsklinik und des Landeskrankenhauses Innsbruck das Vorliegen von psychosomatischen Störungen und Burnout [13]. Dabei wurden etwa ein Viertel der Befragten als „burnout-gefährdet“ angesehen. Ob diese Belastung auch für KollegInnen anderer Fachrichtungen in ähnlicher Weise zutrifft, wurde in dieser Untersuchung nicht erfasst. Im Rahmen einer groß angelegten Studie zum Thema „Bedingt der Eignungstest für Studierende der Humanmedizin (EMS) eine neue ÄrztInnengeneration?" wurde auch der Gesundheitszustand und die Burnout-Symptomatik von KlinikärztInnen unabhängig von der Fachrichtung erhoben.

\section{Methodik}

\section{Studiendesign und Studienpopulation}

Im Rahmen einer groß angelegten Studie zum Thema „Bedingt der Eignungstest für Studierende der Humanmedizin (EMS) eine neue ÄrztInnengeneration?“ wurden alle an der Universitätsklinik und am Landeskrankenhaus Innsbruck tätigen ÄrztInnen ( $n=1090)$ nach persönlicher Vorstellung des Projektes an den jeweiligen unterschiedlichen Einrichtungen per E-Mail kontaktiert. Die Aussendung enthielt sowohl die Studienbeschreibung als auch die Einverständniserklärung und die Onlineversionen der für die Studie herangezogenen Messinstrumente.

Diese Untersuchung wurde von der Ethikkommission der Medizinischen Universität Innsbruck geprüft und für unbedenklich erklärt. Alle Teilnehmer erteilten vor Einschluss in die Studie ihr Einverständnis, die Datenerfassung erfolgte anonym. Insgesamt nahmen 118 ÄrztInnen an der Studie teil, 69 füllten das MBI vollständig aus und konnten somit für die abschließende Berechnung herangezogen werden. 
Tab. 1 Einteilung der Subskalen des Maslach Burnout Inventory (MBI). (Adaptiert von der Einteilung nach Maslach et al. [11])

\begin{tabular}{|l|l|l|l|}
\hline MBI-Subskala & \multicolumn{2}{l}{ Ausprägung } & \\
& Gering & Mäßig & Hoch/stark \\
\hline Emotionale Erschöpfung & $9-19$ & $20-26$ & $27-45$ \\
\hline Depersonalisation & $5-9$ & $10-13$ & $14-25$ \\
\hline Persönliche Erfüllung & $8-29$ & $30-33$ & $34-40$ \\
\hline
\end{tabular}

\section{Messinstrumente}

Neben einem Fragebogen zu soziodemographischen und berufsbezogenen Variablen wurde das MBI eingesetzt.

Das MBI ist der bekannteste Fragebogen zur Untersuchung der Burnout-Symptomatik und außerdem das Untersuchungsinstrument, welches bei den meisten klinischen Studien eingesetzt wird [14]. Über $90 \%$ der Forschungsarbeiten in diesem Bereich verwenden das MBI [15]. Es eignet sich vor allem für Personen, die im Dienstleistungsbereich oder im Gesundheitswesen arbeiten und wurde auch speziell für diese Zielgruppe entwickelt [16]. Die Entwicklung der deutschen Version des Maslach Burnout Inventory, das MBI-D, lässt sich auf Büssing und Perrar [17] zurückführen.

Das MBI besteht aus drei Subskalen. Diese erfassen die emotionale Erschöpfung, die Depersonalisation und die persönliche Erfüllung. Depersonalisation ist dabei charakterisiert durch emotionale Distanzierung zu den behandelten Patienten sowie zunehmende Gleichgültigkeit und eine zynische Einstellung gegenüber diesen Personen [18]. Hohe Werte in den Bereichen Emotionale Erschöpfung und Depersonalisation sowie niedrige Werte im Bereich Persönliche Erfüllung gehen mit einer größeren Burnout-Gefährdung einher [19]. Diese drei Komponenten werden anhand 24 Items erfasst und ausgewertet. Die Antwortmöglichkeiten für die Befragten bestehen aus einer siebenstufigen Skala und reichen dabei von $0=$ nie bis $7=$ täglich. Die deutsche Version des MBI weist eine gute interne Konsistenz für die Subskala Emotionale Erschöpfung (Cronbachs alpha von 0,82) und zufriedenstellende Werte für die Skalen Persönliche Erfüllung $(\alpha=0,75)$ und Depersonalisation $(\alpha=0,67)$ auf [20].

Maslach, Jackson und Leiter [11] gaben für die drei Subskalen des MBI eine Einteilung in die Stufen „gering“, „mäßig“ und „stark“ an. Nach Umrechnung der siebenstufigen Original-Antwortskala auf die hier verwendete fünfstufige Antwortskala führt diese Klassifikation zu der in Tab. 1 dargestellten Einteilung. Ferner wurde in Anlehnung an die Studie von Denton et al. [21] basierend auf den Kombinationen der drei MBISubskalen eine Einstufung in drei Risikogruppen vorgenommen:

1. hohes Burnout-Risiko, falls mindestens zwei der drei MBI-Subskalen die Ausprägung „stark“ aufwie- sen (bzw. bei der umgepolten Subskala Persönliche Erfüllung die Ausprägung „gering“) und die dritte Subskala zumindest den Grad „mittel“;

2. mittleres Burnout-Risiko, falls zwei der drei MBISubskalen die Ausprägung „stark“ aufwiesen und die dritte Subskala den Grad „gering“ (bei der Subskala Persönliche Erfüllung wiederum genau umgekehrt) oder eine der Subskalen die Ausprägung „stark" und die beiden anderen die Ausprägung „mittel“;

3. geringes bzw. kein Burnout-Risiko bei allen anderen Kombinationen der MBI-Subskalen.

\section{Statistik}

Alle statistischen Analysen wurden mit dem Statistikprogramm SPSS, Version 24 durchgeführt. Neben Methoden der deskriptiven Statistik wurden die folgenden Verfahren der schließenden Statistik verwendet: Fishers exakter Test zum Vergleich zweier Gruppen bezüglich dichotomer Variablen, der Mann-Whitney U-Test für den Vergleich zweier Gruppen bezüglich der MBI-Subskalen, da zwei der drei Subskalen signifikante Abweichungen von einer Normalverteilung aufwiesen (Überprüfung mittels Shapiro-Wilk-Test). Alle statistischen Tests wurden auf einem Signifikanzniveau von $\alpha=0,05$ durchgeführt.

\section{Ergebnisse}

\section{Beschreibung der Stichprobe}

Insgesamt wurden alle 1090 KlinikärztInnen per mail zu dieser Studie eingeladen, 118 (11\%) nahmen tatsächlich an dieser Untersuchung teil. 69 (58\%) davon füllten den MBI vollständig aus und konnten damit in die nachfolgende Untersuchung eingeschlossen werden. Eine Übersicht über wichtige soziodemografische und berufsbezogene Charakteristika der Stichprobe findet sich in Tab. 2. Die Studienteilnehmer hatten ein Durchschnittsalter von 37 Jahren, etwa drei Fünftel von ihnen waren weiblich. Die teilnehmenden KlinikärztInnen arbeiteten im Durchschnitt $54 \mathrm{~h}$ pro Woche und leisteten im Mittel 1,8 Wochenenddienste pro Monat. Knapp zwei Drittel der ÄrztInnen (63\%) gaben an, vorwiegend im Versorgungsbereich tätig zu sein, während die übrigen sowohl wissenschaftlich als auch in der Versorgung (34\%) oder vorwiegend wissenschaftlich (3\% bzw. 2 ÄrztInnen) tätig waren. Weitere Angaben können der Tab. 2 entnommen werden.

\section{Ergebnisse zur Burnout-Symptomatik}

Eine Übersicht über die Ergebnisse der Erhebung der Burnout-Symptome mit dem MBI findet sich in Abb. 1 und Tab. 3. Nach der oben beschriebenen Einteilung der MBI-Subskalen in drei Stufen fanden sich bei $13 \%$ der Ärzte starke und bei weiteren $42 \%$ mäßige Symptome von emotionaler Erschöpfung, während die 
Tab. 2 Soziodemografische und berufsbezogene Variablen $(N=69)$

\begin{tabular}{|c|c|c|}
\hline Variable & Kategorie & $\begin{array}{l}N(\%) \text { oder Mittel- } \\
\text { wert } \pm \text { SD }\end{array}$ \\
\hline Alter & Jahre & $36,8 \pm 6,5$ \\
\hline \multirow[t]{2}{*}{ Geschlecht } & Männlich & $27(39 \%)$ \\
\hline & Weiblich & $42(61 \%)$ \\
\hline \multirow[t]{2}{*}{ Familienstand } & Feste Partnerschaft & $53(77 \%)$ \\
\hline & Ohne Partner & $16(23 \%)$ \\
\hline \multirow[t]{2}{*}{ Kinder } & Keine & $34(53 \%)$ \\
\hline & 1 oder mehr & $30(47 \%)$ \\
\hline \multirow[t]{4}{*}{ Fachrichtung } & Operative Fächer & $20(31,7 \%)$ \\
\hline & Nicht-operative Fächer & $16(25,4 \%)$ \\
\hline & Psychiatrie/Neurologie & $10(15,9 \%)$ \\
\hline & Anästhesie & $17(27,0 \%)$ \\
\hline \multirow[t]{2}{*}{ Tätigkeitsbereich } & Vorwiegend Versorgung & $43(63,2 \%)$ \\
\hline & $\begin{array}{l}\text { Vorwiegend Wissen- } \\
\text { schaft oder Wissen- } \\
\text { schaft+Versorgung }\end{array}$ & $25(36,8 \%)$ \\
\hline \multirow[t]{2}{*}{ Anstellung } & Bund & $30(43,5 \%)$ \\
\hline & Land & $39(56,5 \%)$ \\
\hline \multirow[t]{2}{*}{ Dienststellung } & Facharzt & $26(42,6 \%)$ \\
\hline & In Ausbildung & $35(57,4 \%)$ \\
\hline Berufsjahre & Jahre & $8,3 \pm 6,0$ \\
\hline Arbeitszeit pro Woche & Stunden & $54,2 \pm 12,6$ \\
\hline Wochenenddienste & Anzahl & $1,8 \pm 1,3$ \\
\hline
\end{tabular}

restlichen 45 \% höchstens geringe Symptome zeigten. Jeweils etwas höhere Werte für eine starke (19\%) bzw. mäßige (44\%) Symptomatik zeigte sich bei der MBISubskala Depersonalisation. Hinsichtlich der persönlichen Erfüllung, bei der niedrige Werte als BurnoutGefährdung anzusehen sind, fand sich bei $43 \%$ der teilnehmenden Ärzte eine Einstufung als gering (ungünstigste Ausprägung) und bei weiteren $39 \%$ als mäßig.

Bei einem Vergleich der MBI-Scores von Klinikärzten, die hauptsächlich im Versorgungbereich tätig sind, mit denjenigen von Ärzten, die auch oder vorwiegend wissenschaftlich arbeiten, zeigten sich keine Unterschiede in der emotionalen Erschöpfung und nur geringfügige, statistisch nicht signifikante Unterschiede in der Depersonalisation (mit etwas günstigeren Werten bei wissenschaftlich tätigen Ärzten). Bei der persönlichen Erfüllung fanden sich hingegen deutliche Unterschiede zugunsten wissenschaftlich arbeitender Klinikärzte (Mittelwert von 3,92 $\pm 0,33$ vs. $3,64 \pm 0,42, \mathrm{Z}=2,7, p=0,007$, Mann-Whitney U-Test). Details finden sich in Abb. 2. Auch in Bezug auf die Einteilung nach Ausprägungsgrad ergaben sich signifikante Unterschiede in der persönlichen Erfüllung: $53,5 \%$ der vorwiegend im Versorgungsbereich tätigen Ärzte, aber nur $28 \%$ der wissenschaftlich arbeitenden Ärzte zeigten eine geringe persönliche Erfüllung ( $p=$ 0,048, Fishers exakter Test). Ein Vergleich der Ärzte nach ihrer Fachrichtung zeigte keine signifikanten Unterschiede in den Subskalen des MBI. Ebenso fand

Abb. 1 Burnout-Symptomatik bei Klinikärzten (MBI) (MBI Maslach Burnout Inventory)

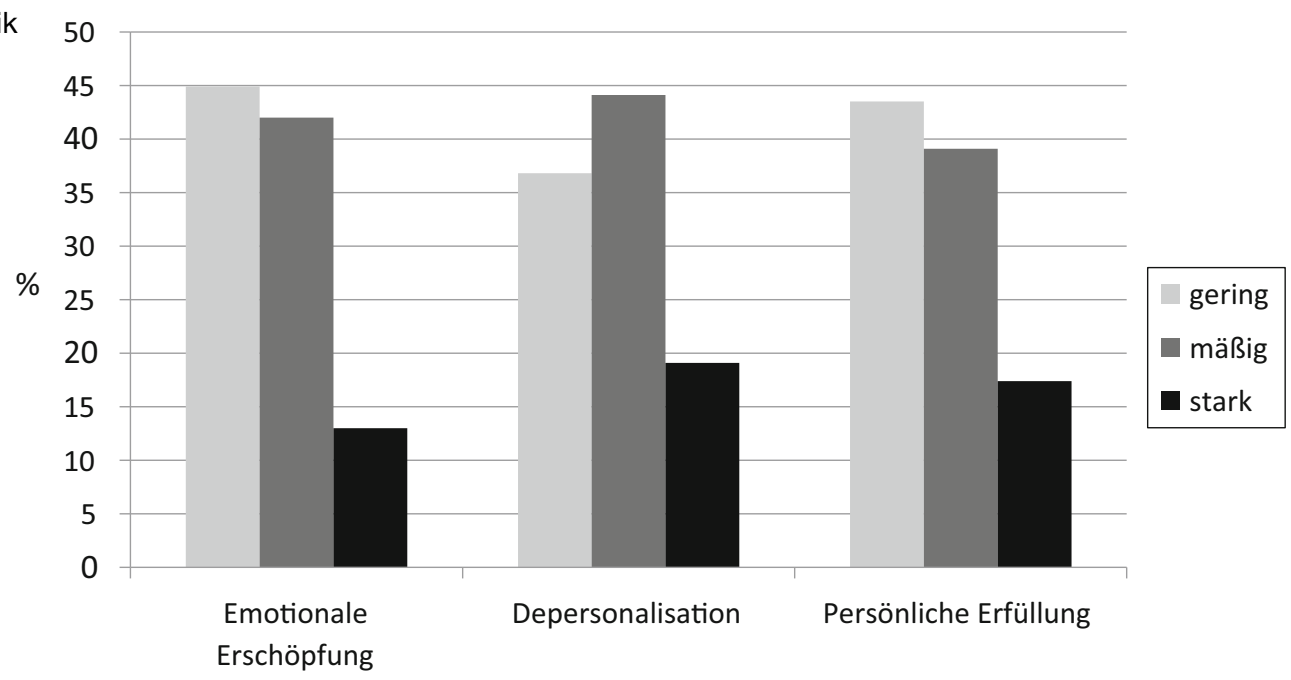

Tab. 3 Maslach Burnout Inventory (MBI) - Ergebnisse für die Gesamtstichprobe ( $N=69)$

\begin{tabular}{|c|c|c|c|c|}
\hline \multirow[t]{2}{*}{ MBI Subskalen } & \multirow[t]{2}{*}{ Mittelwert $\pm \mathrm{SD}^{\mathrm{a}}$} & \multicolumn{3}{|c|}{ Grad der Ausprägung (nach Maslach) } \\
\hline & & Gering & Mäßig & Stark/hoch \\
\hline Emotionale Erschöpfung & $2,37 \pm 0,61$ & $31(44,9 \%)$ & $29(42,0 \%)$ & $9(13,0 \%)$ \\
\hline Depersonalisation & $2,14 \pm 0,58$ & $25(36,8 \%)$ & $30(44,1 \%)$ & $13(19,1 \%$ \\
\hline Persönliche Erfüllung & $3,74 \pm 0,41$ & $30(43,5 \%)$ & $27(39,1 \%)$ & $12(17,4 \%)$ \\
\hline
\end{tabular}


Abb. 2 Burnout-Symptomatik in Abhängigkeit vom Tätigkeitsbereich ( ${ }^{*}$ Signifikant größere persönliche Erfüllung bei Ärzten im Bereich Wissenschaft + Versorgung)

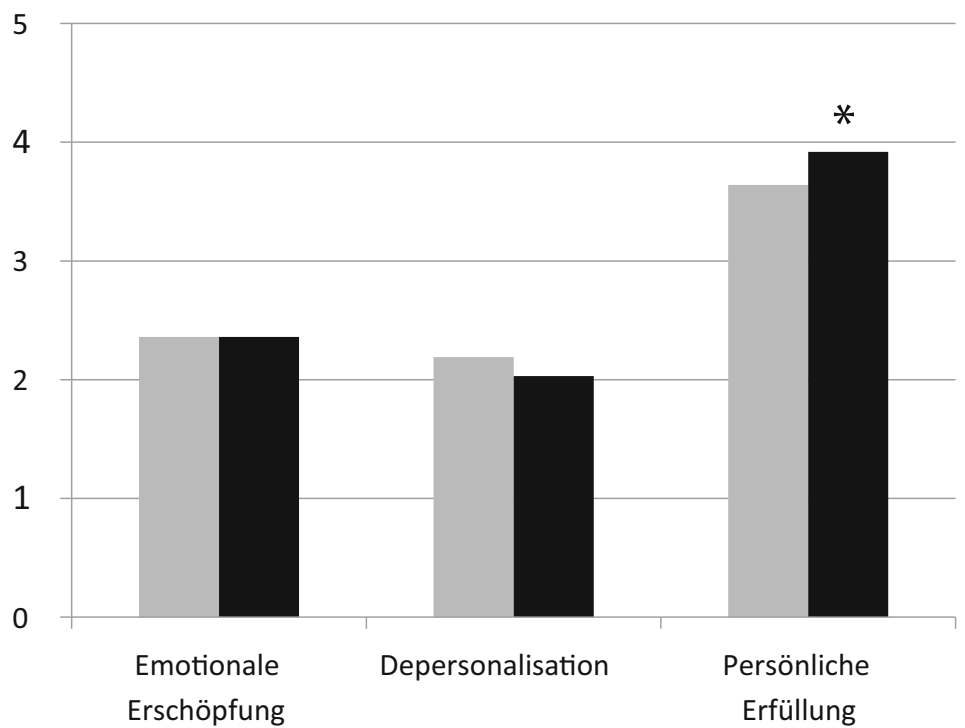

Hauptsächlich Versorgung

Wissenschaft +Versorgung

Tab. 4 Studienteilnehmer mit Burnout-Risiko (Kombination von emotionaler Erschöpfung, Depersonalisation und persönlicher Erfüllung)

\begin{tabular}{|c|c|c|c|c|c|c|}
\hline \multirow[t]{2}{*}{ Burnout-Risiko } & \multicolumn{3}{|c|}{ Kombination im MBI } & \multirow[t]{2}{*}{ Anzahl } & \multirow[t]{2}{*}{ Prozent } & \multirow[t]{2}{*}{ Insgesamt } \\
\hline & $\begin{array}{l}\text { Emotionale } \\
\text { Erschöpfung }\end{array}$ & Depersonalisation $^{\mathrm{a}}$ & Persönliche Erfüllung ${ }^{\mathrm{a}}$ & & & \\
\hline \multirow[t]{3}{*}{ Hoch } & Hoch & Hoch & Gering & 3 & 4,4 & \multirow[t]{3}{*}{$6(8,8 \%)$} \\
\hline & Hoch & Hoch & Mittel & 1 & 1,5 & \\
\hline & Hoch & Mittel & Gering & 2 & 2,9 & \\
\hline \multirow[t]{4}{*}{ Mittel } & Mittel & Mittel & Gering & 4 & 5,9 & \multirow[t]{4}{*}{$8(11,8 \%)$} \\
\hline & Mittel & Hoch & Mittel & 1 & 1,5 & \\
\hline & Hoch & Gering & Gering & 2 & 2,9 & \\
\hline & Gering & Hoch & Gering & 1 & 1,5 & \\
\hline $\begin{array}{l}\text { Gering/ } \\
\text { kein Risiko }\end{array}$ & \multicolumn{3}{|c|}{ Alle anderen Kombinationen } & 54 & 79,4 & $54(79,4 \%)$ \\
\hline Gesamt & \multicolumn{3}{|l|}{-} & 68 & 100,0 & $100,0 \%$ \\
\hline
\end{tabular}

sich kein signifikanter Zusammenhang zwischen der Anzahl der Berufsjahre und der Burnout-Symptomatik (Spearman Rangkorrelationen, $\mathrm{r}_{\mathrm{s}}<0,2, p>0,1$ ).

\section{Burnout-Risiko}

Bezüglich der oben beschriebenen Einteilung nach Burnout-Risiko aufgrund der Kombinationen der drei MBI-Subskalen ergab sich für 8,8\% der KlinikärztInnen ein hohes Risiko. Diese ÄrztInnen waren durch starke emotionale Erschöpfung, starke oder mittelgradige Depersonalisation und geringe bzw. in einem Fall mäßige persönliche Erfüllung charakterisiert. Weitere $11,8 \%$ der teilnehmenden ÄrztInnen zeigten ein mäßiges Burnout-Risiko. Die jeweils aufgetretenen Kombinationen der Ausprägungsgrade von emotionaler Erschöpfung, Depersonalisation und eingeschränkter persönlicher Erfüllung sind in Tab. 4 wiedergegeben. Insgesamt hatten damit 20,6\% der teilnehmenden Klinikärzte ein mittleres oder hohes Burnout-Risiko.
Ein Vergleich nach Tätigkeitsbereich zeigt, dass 12 $(27,9 \%)$ der hauptsächlich in der Versorgung tätigen Ärzte, aber nur $2(8,3 \%)$ der teilweise oder hauptsächlich wissenschaftlich arbeitenden Ärzte ein mittleres oder hohes Burnout-Risiko hatten. Dieser Unterschied erreichte allerdings nur tendenzielle Signifikanz ( $p=$ 0,069, Fishers exakter Test).

\section{Limitationen}

Die Limitationen dieser Untersuchung sind die geringe Responsrate sowie die Tatsache, dass Selbstbewertungsfragebögen verwendet wurden. Diese Querschnittsuntersuchung - Untersuchung gibt auch keine Information über kausale Zusammenhänge im Rahmen der Burnout-Entwicklung bei ÄrztInnen. 


\section{Fazit für die Praxis}

- Die erste große Untersuchung über Burnout-Symptomatik bei ÄrztInnen wurde 2012 von Shanafelt et al. [9] publiziert. Hier zeigte sich in einer Population von 7288 US.-ÄrztInnen bei $37,9 \%$ eine hohe emotionale Erschöpfung, bei 29,4 \% eine hoher Grad an Depersonalisation und bei 12,4 \% ein geringes Ausmaß an persönlicher Erfüllung. Verglichen mit unseren Ergebnissen zeigt sich hier eine deutlich höhere Ausprägung von hoher emotionaler Erschöpfung (37,9 \% vs. $13 \%)$ und starker Depersonalisation (29,4\% vs. $19,1 \%)$ aber ein deutlich geringeres Ausmaß an geringer persönlicher Erfüllung (12,4 \% vs. $43,5 \%)$. Zum annähernd selben Ergebnis kommt auch eine italienische Studie aus dem Jahr 2000 von Grassi und Magnani [22]. Eine Studie aus British Columbia zeigt in ihren Ergebnissen auch mit 80 \% (vs. $55 \%$ ) deutlich höhere Werte in Bezug auf mäßige und starke emotionale Erschöpfung, ein annähernd gleich ausgeprägtes Ausmaß an mäßig und stark ausgeprägter Depersonalisation (61 \% vs. $63,2 \%$ ) und wiederum eine geringere Ausprägung von mäßiger und geringer persönlicher Erfüllung (44 \% vs. 82,6 \%) [23]. Es bestätigt sich also, gleichermaßen, dass sowohl die Prävalenz von Burnout im Gesamten, wie auch die Prävalenz der einzelnen Burnout-Dimensionen zwischen den einzelnen Ländern variieren [24]. Es sollte allerdings beachtet werden, dass die Beurteilung des Ausmaßes der Burnout-Symptomatik nicht in allen Studien in derselben Weise erfolgt ist, wodurch die Vergleichbarkeit der Ergebnisse erschwert wird.

- In der Literatur finden sich bezüglich Österreich nur vereinzelt Arbeiten zum Thema Burnout bei ÄrztInnen. 2006 untersuchte Kinzl et al. [13] die BurnoutBelastung bei 89 Intensivmedizinern und beschrieb eine Burnout-Gefährdung von $25 \%$ in dieser speziellen Fachgruppe. 2011 wurde von Hofmann im Auftrag der Österreichischen Ärztekammer (ÖÄK) eine Online-Untersuchung der heimischen Ärzteschaft durchführte. Bislang ist daraus nur bekannt, dass $54 \%$ der Befragten sich in unterschiedlichen Phasen des Burnouts - von der relativ "harmlosen“ Phase I bis hin zur behandlungsbedürftigen Phase III befinden [25]. Unserer Untersuchung zeigt nun differenzierter, dass basierend auf der Kombination der drei MBI-Subskalen 20,6 \% der teilnehmenden KlinikärztInnen ein mittleres oder hohes Burnout-Risiko aufweisen. Da Betroffene in der Regel auch in ihrer Leistungsfähigkeit eingeschränkt sind [10] kann auch eine adäquate Patientenversorgung durch die Betroffenen gefährdet sein, was die Bedeutung dieser Thematik noch unterstreicht.

- In der Literatur wird der Einfluss von Soziodemographischen Variablen auf die Burnout Entwicklung vielfach widersprüchlich diskutiert. Studien aus den USA zeigen zum Beispiel eine höhere Burnoutge- fährdung unter Ärztinnen während Untersuchungen in Europa ein höheres Burnoutrisiko bei Ärzten beschreiben [24]. Diese Widersprüchlichkeit ist sicher darin begründet, dass die Untersuchungen in verschiedenen Ländern mit unterschiedlichen kulturellen Hintergründen und Arbeitsbedingungen wie auch an verschiedenen ÄrztInnengruppen durchgeführt wurden.

- In unserer Untersuchung ergab sich beim Vergleich der MBI-Scores hinsichtlich Alter, Geschlecht, Familienstand, Fachrichtung, Berufsjahre, Wochenarbeitszeit und Anzahl der Wochenenddienste kein signifikanter Einfluss. Ein neues und überraschendes Ergebnis ist die Tatsache, dass wissenschaftliches Arbeiten sich als protektiver Faktor im Rahmen der Burnout-Gefährdung bei Klinikärzten erwies. Bei der persönlichen Erfüllung zeigten sich deutliche Unterschiede zugunsten wissenschaftlich arbeitender KlinikärztInnen. Wissenschaftliches Arbeiten scheint daher nicht als Mehrbelastung das Burnoutrisiko zu erhöhen, sondern im Gegenteil diesbezüglich einen protektiven Effekt auszuüben. Erklärbar ist dieses Ergebnis wohl durch die Tatsache, dass wissenschaftliches Arbeiten die Selbstwirksamkeit erhöht und den Erhalt von Belohnung im Austausch von Leistung und Gegenleistung fördert und damit das Selbstwertgefühl erhöht. Es bietet also einen Gegenpol zu den sozialen Stressoren die in der modernen Arbeitswelt häufig und wiederkehrend auftreten und die Burnoutgefährdung erhöhen [26]. In den letzten 20 Jahren haben sich einfach die Arbeitsbedingungen der ÄrztInnen deutlich verändert. Die Arbeitsautonomie sowie der gesellschaftliche Status allgemein haben abgenommen, dafür ist die Arbeitsbelastung stetig angestiegen [27].

- Die in der vorliegenden Untersuchung aufgezeigte Burnout-Gefährdung von KlinikärztInnen verdient ernst genommen zu werden. Die Erforschung der dazu führenden Ursachen sollte intensiviert werden, um dann in Folge spezifische Präventivmaßnahmen entwickeln und etablieren zu können. Zusätzlich bedarf es auch der Entwicklung und Bereitstellung spezieller Therapieangebote, die den Kriterien von Leitlinien entsprechend fundierte Empfehlungen beinhalten.

Open access funding provided by University of Innsbruck and Medical University of Innsbruck.

Interessenkonflikt I. Kurzthaler, G. Kemmler und W. W. Fleischhacker geben an, dass kein Interessenkonflikt besteht.

Open Access Dieser Artikel wird unter der Creative Commons Namensnennung 4.0 International Lizenz (http:// creativecommons.org/licenses/by/4.0/deed.de) veröffentlicht, welche die Nutzung, Vervielfältigung, Bearbeitung, Verbreitung und Wiedergabe in jeglichem Medium und Format erlaubt, sofern Sie den/die ursprünglichen Autor(en) und die Quelle ordnungsgemäß nennen, einen Link zur Creative Commons Lizenz beifügen und angeben, ob Änderungen vorgenommen wurden. 


\section{Literatur}

1. Köksal Baltaci. Wien steuert auf Ärztemangel zu „Die Presse“, Print-Ausgabe, 08.11.2016.

2. Kauffeld S, Carsten C, Schermuly S. Arbeitszufriedenheit und Arbeitsmotivation. In: Arbeits-, Organisationsund Personalpsychologie für Bachelor. Berlin Heidelberg: Springer;2014.

3. Cassel CK, Jain SH. Assessing individual physician performance. Does measurement supress motivation? JAMA. 2012;307(24):2595-6.

4. Schaufeli WT, Leiter MP, Maslach C. Burnout: 35 years of research and practice. Career DevInt. 2009;14(3):204-20.

5. Maslach C, Schaufeli WT, Leiter MP. Job burnout. Annu Rev Psychol. 2001;52:397-422. doi:10.1146/annurev.psych. 52.1.397.

6. Burisch M. Das Burnout-Syndrom: Theorie der inneren Erschöpfung, 5. Aufl. Berlin Heidelberg: Springer; 2014.

7. Krollner B. ICD-Code 2014. http:/ / www.icd-code.de. Zugegriffen: 11. März2014.

8. Känel R. Das Burnout-Syndrom: eine medizinische Perspektive. Praxis. 2008;97:477-87.

9. Shanafelt TD, Boone S, Tan L, Dyrbye LN, Sotile W, Satele D, West CP, Sloan J, Oreskovich MR. Burnout and satisfaction with work-life balance among US physicians relative to the general US population. Arch Intern Med. 2012;172(18):1377-85.

10. Michaelsen A, Hillert A. Burn-out in Anästhesie und Intensivmedizin. Anaesthesist. 2011;60:31-8.

11. Maslach C, Jackson SE, Leiter MP. The Maslach burnout inventory, 3. Aufl. Palo Alto: Consulting Psychologists Press; 1996.

12. Reimann S. Die medizinische Sozialisation. Wiesbaden: Springer;2013.doi:10.1007/978-3-531-19847-7_1.

13. Kinzl JF, Traweger C, Biebl W, Lederer W. Burnout und Belastungsstörungen bei Intensivmedizinern. Dtsch Med Wochenschr. 2006;131:2461-4.

14. Schmiedel V. Burnout: Wenn Arbeit, Alltag \& Familie erschöpfen, welche körperlichen Untersuchungen Ihnen jetzt weiterhelfen, wie sie aus der Stressspirale aussteigen. Stuttgart:Trias; 2010.

15. Buehrsch NC, Mewes AUJ, Knorr F, Zoller T, Kunkel J, Urbanek C, Kleinjung F, Guckelberger O, Tegethoff K, Peters O. Children lower the probability of bournout in physicians. JDepress Anxiety. 2011; doi:10.4172/jda.1000108.

16. Prins JT, Hoekstra-Weebers JEHM, Gazendam-Donofrio SM, Dillingh GS, Bakker AB, Huisman M, et al. Burnout and engagement among resident doctors in the Netherlands: a national study. Med Educ. 2010;44(3):236-47.

17. Büssing A, Perrar KM. Die Messung von Burnout. Untersuchung einer deutschen Fassung des Maslach Burnout Inventory (MBI-D). Diagnostica. 1992;38(4):328-53.

18. Maslach C, Schaufeli WB, Leiter MP. Job burnout. Annu Rev Psychol. 2001;52:397-422.

19. Hedderich I. Burnout: Ursachen, Formen, Auswege. München:C.H. Beck; 2009 .

20. Enzmann D, Kleiber D. Helfer-Leiden: Stress und Burnout in psychosozialen Berufen. Heidelberg: Asanger; 1989.

21. Denton DA, Newton JT, Bower EJ. Occupational burnout and work engagement: a national survey of dentists in the United Kingdom. Br Dent J. 2008;205:E13.

22. Grassi L, Magnani K. Psychiatric morbidity and burnout in the medical profession: an Italian study of general practitioners and hospital physicians. Psychother Psychosom. 2000;69(6):329-34.

23. Lee RT, Seo B, Hladkyj S, Lovell BL, Schwartzmann L. Correlates of physician burnout across regions and specialties: a meta-analysis. Hum Resour Health. 2013;11:48. doi:10. 1186/1478-4491-11-48.

24. Kumar S. Burnout and doctors: prevalence, prevention and intervention. Healthcare (Basel). 2016;4:3-E37. doi:10. 3390/healthcare4030037.

25. Mayerhofer R. Ergebnisse der Burnoutstudie. Österreichische Ärztezeitung 25.04.2011; Nr. 8.

26. Siegrist J. Burn-out und Arbeitswelt. Psychotherapeut. 2013;58:110-6.

27. Linzer M, Visser MRM, Oort FJ, et al. Predicting and preventing physician burnout: results from the United States and the Netherlands. Am J Med. 2001;111:170-5. 